\title{
Curvilinear optical forces
}

\author{
Andrey Novitsky \\ Department of Theoretical Physics and Astrophysics, Belarusian State University, Nezavisimosti Avenue 4, 220030 Minsk, Belarus
}

(Received 26 June 2015; published 14 August 2015)

\begin{abstract}
Accelerating a light beam makes a particle move along a curvilinear trajectory. We show that the curvature of the trajectory causes a special kind of nonconservative force, which is called a curvilinear force. We obtain the expression for this force acting on a spherical particle in the Rayleigh approximation and determine its value for several types of accelerating fields including Bessel, Airy, and arbitrary-trajectory beams. We anticipate applications of the curvilinear forces in optical micromanipulation with accelerating beams.
\end{abstract}

DOI: 10.1103/PhysRevA.92.023823

PACS number(s): 42.50.Wk, 42.25.Fx

\section{INTRODUCTION}

The peculiar properties of accelerating electromagnetic beams were first revealed less than ten years ago in Refs. [1,2]. The Airy beam was the first accelerating light beam to be realized. The idea of this beam stems from the pioneering paper by Berry and Balazs on nondiffracting wave packets in quantum mechanics [3]. The nondiffracting Airy beam has lobes of parabolic shape (parabolic "channels"), which can be used for dragging particles by nongradient (nonconservative) optical forces. Going beyond the paraxial approximation, it is necessary to deal with the solutions of the Helmholtz wave equation to describe the behavior of the Airy beams [4-6]. A nonparaxial Airy beam does not keep its spatial structure upon propagation and loses its extraordinary features on the short distance from the beam's source.

To improve the concept of the diffraction-free nonparaxial accelerating beam, Kaminer et al. recently proposed [7] the theoretical method of generation of a beam (similar to the Bessel field) with circular channels by superposition of the plane waves. The authors of Ref. [8] derived the conditions for optimal control of the general ballistic trajectory of the Airy beams. Generally speaking, accelerating light beams with arbitrary trajectories are diffractive. An arbitrary trajectory of a Bessel-like beam can be formed by an ensemble of rays on the cones with apexes lying on a preassigned curve $[9,10]$. All light beams accelerating along a convex trajectory [11] possess universal properties following from the catastrophe theory [12]. The propagation characteristics of the accelerating beams can be understood using the traveling-wave approach as in Ref. [13].

The concept of optical micromanipulation with accelerating light beams was proposed for the first time in Ref. [14]. There is also a number of theoretical papers on the optical forces produced by accelerating fields. Along with investigations of the gradient forces in the Rayleigh approximation $[15,16]$, the Mie scattering theory paves the way for the optical manipulation of large spherical particles using Airy beams [17,18].

In this work, we analyze the set of optical forces exerted by accelerating light beams and introduce the curvilinear and polarization forces together with the well-known intensity gradient and phase gradient forces. The paper is organized as follows. In Sec. II, we define five optical forces in the Rayleigh approximation: electrical gradient, magnetic gradient, phase gradient, polarization, and curvilinear optical forces. In Sec. III, we consider the curvilinear optical forces for nondiffracting Bessel and Airy beams. Section IV is devoted to the investigation of the optical forces produced by the light beams with arbitrarily designed channels. Finally, Sec. V concludes the paper.

\section{OPTICAL FORCES VIA AN ACCELERATING LIGHT BEAM}

We consider small spherical nonmagnetic particles (dielectric permittivity $\varepsilon$ ) in vacuum with radii $a$ that are much smaller than the radiation wavelength $\lambda$. The time-averaged optical force acting on the Rayleigh particle in the monochromatic electromagnetic field can be written using electric $\mathbf{p}$ and magnetic $\mathbf{m}$ dipole moments as [19]

$$
\mathbf{F}=\frac{1}{2} \operatorname{Re}\left(\nabla\left(\mathbf{p} \mathbf{E}^{*}\right)+\nabla\left(\mathbf{m} \stackrel{\downarrow}{*}^{*}\right)-\frac{2 k_{0}^{4}}{3} \mathbf{p} \times \mathbf{m}^{*}\right),
$$

where $\downarrow$ points out the quantities subjected to differentiation, and $\mathbf{E}(\mathbf{r})$ and $\mathbf{H}(\mathbf{r})$ are the electric and magnetic field strengths, respectively, of the incident wave taken at the particle's center. Electric and magnetic polarizabilities are introduced as the proportionality coefficients between the dipole moment and the field, $\mathbf{p}=\alpha_{e} \mathbf{E}$ and $\mathbf{m}=\alpha_{m} \mathbf{H}$. We briefly show how the electric and magnetic polarizabilities of a nonmagnetic sphere can be calculated in the Rayleigh approximation. In general, the polarizabilities are expressed in terms of the Mie coefficients $a_{1}$ and $b_{1}$ as

$$
\alpha_{e}=\frac{3 i a_{1}}{2 k_{0}^{3}}, \quad \alpha_{m}=\frac{3 i b_{1}}{2 k_{0}^{3}},
$$

where $k_{0}=\omega / c=2 \pi / \lambda$ is the wave number in vacuum, $\omega$ is the circular frequency, and $c$ is the speed of light in vacuum. The Mie coefficients [20]

$$
\begin{aligned}
& a_{1}=\frac{n^{2} j_{1}(n s)\left[s j_{1}(s)\right]^{\prime}-j_{1}(s)\left[n s j_{1}(n s)\right]^{\prime}}{n^{2} j_{1}(n s)\left[s h_{1}^{(1)}(s)\right]^{\prime}-h_{1}^{(1)}(s)\left[n s j_{1}(n s)\right]^{\prime}}, \\
& b_{1}=\frac{j_{1}(n s)\left[s j_{1}(s)\right]^{\prime}-j_{1}(s)\left[n s j_{1}(n s)\right]^{\prime}}{j_{1}(n s)\left[s h_{1}^{(1)}(s)\right]^{\prime}-h_{1}^{(1)}(s)\left[n s j_{1}(n s)\right]^{\prime}}
\end{aligned}
$$

can be reduced to the rational functions of the small parameter $s=k_{0} a \ll 1$ for the Rayleigh objects $(n=\sqrt{\varepsilon}$ is the refractive index), when the series expansion of the spherical Bessel $j_{1}$ and Hankel $h_{1}$ functions of the first order is applied. Thus, we 
get to

$$
\alpha_{e}=\frac{\alpha_{0 e} / k_{0}^{3}}{1-2 \mathrm{i} \alpha_{0 e} / 3}, \quad \alpha_{m}=\frac{\alpha_{0 m} / k_{0}^{3}}{1-2 \mathrm{i} \alpha_{0 m} / 3},
$$

where the normalized static electric and magnetic polarizabilities equal

$$
\alpha_{0 e}=\frac{\varepsilon-1}{\varepsilon+2} s^{3}, \quad \alpha_{0 m}=\frac{\varepsilon-1}{30} s^{5} .
$$

For the small values of $\alpha_{0 e}$ and $\alpha_{0 m}$, the polarizabilities given by Eq. (4) read

$$
\alpha_{e} \approx \frac{\alpha_{0 e}}{k_{0}^{3}}+\frac{2 \mathrm{i} \alpha_{0 e}^{2}}{3 k_{0}^{3}}, \quad \alpha_{m} \approx \frac{\alpha_{0 m}}{k_{0}^{3}}+\frac{2 \mathrm{i} \alpha_{0 m}^{2}}{3 k_{0}^{3}} .
$$

In the case of lossless dielectric Rayleigh particles, the real part $\operatorname{Re} \alpha_{e} \sim s^{3}$ is responsible for the gradient forces, while the imaginary part $\operatorname{Im} \alpha_{e} \sim s^{6}$ is responsible for the nonconservative forces. Since the magnetic polarizability $\alpha_{m} \approx \operatorname{Re} \alpha_{m} \sim s^{5}$ is comparable with the imaginary part of the electric polarizability, we consider the force due to the gradient of the magnetic field as well. The interaction of the electric and magnetic dipole moments is described by the quantity $\operatorname{Re}\left(\mathbf{p} \times \mathbf{m}^{*}\right) \sim \operatorname{Re} \alpha_{e} \operatorname{Re} \alpha_{m} \sim s^{8}$ [see Eq. (1)] and can be omitted. Then, the optical force is of the form

$$
\mathbf{F}=\frac{1}{2} \operatorname{Re}\left(\alpha_{e} \nabla\left(\mathbf{E} \mathbf{E}^{\downarrow}\right)+\alpha_{m} \nabla\left(\mathbf{H} \stackrel{\mathbf{H}}{*}^{\downarrow}\right)\right) .
$$

Let the incident field be the accelerating light beam with the channel defined by the in-plane curvilinear trajectory $\xi(x, z)=$ const. We look for the optical forces, which can stably trap and drag a spherical particle within a channel. We associate the orthogonal curvilinear coordinate system with the moving particle assuming $(\xi(x, z), \eta(x, z), y)$. Basis vectors $\mathbf{e}_{\xi}(\xi, \eta)$, $\mathbf{e}_{\eta}(\xi, \eta)$, and $\mathbf{e}_{y}$ are normalized and orthogonal, i.e., $\left|\mathbf{e}_{\xi}\right|=$ $\left|\mathbf{e}_{\eta}\right|=\left|\mathbf{e}_{y}\right|=1$ and $\mathbf{e}_{\xi} \mathbf{e}_{\eta}=\mathbf{e}_{\xi} \mathbf{e}_{y}=\mathbf{e}_{\eta} \mathbf{e}_{y}=0$. In this section, we assume that the electric field in a curvilinear coordinate system reads

$$
\begin{aligned}
\mathbf{E}(\xi, \eta, y) & =e^{\mathrm{i} \Phi(\xi, \eta, y)} \mathcal{E}(\xi, \eta, y), \\
\mathcal{E} & =\mathcal{E}_{\xi} \mathbf{e}_{\xi}+\mathcal{E}_{\eta} \mathbf{e}_{\eta}+\mathcal{E}_{y} \mathbf{e}_{y},
\end{aligned}
$$

where $\Phi$ is the phase and $\mathcal{E}$ is the complex amplitude vector. When the particle approaches the stable channel $\xi=\xi(x, z)$, the $\xi$ component of the optical force should vanish.

Using the relationships

$$
\nabla\left(\mathbf{E} \stackrel{\llcorner}{*}^{*}\right)=\nabla\left(\mathcal{E} \stackrel{\downarrow}{\mathcal{E}^{*}}\right)-\mathrm{i}|\mathbf{E}|^{2} \nabla \Phi
$$

and

$$
\nabla\left(\mathcal{E} \stackrel{\downarrow}{\mathcal{E}}^{*}\right)=\mathcal{E}_{\xi} \nabla \mathcal{E}_{\xi}^{*}+\mathcal{E}_{\eta} \nabla \mathcal{E}_{\eta}^{*}+\mathcal{E}_{y} \nabla \mathcal{E}_{y}^{*}+\nabla\left[\mathcal{E}\left(\mathcal{E}_{\xi}^{*} \stackrel{\downarrow}{\mathbf{e}_{\xi}}+\mathcal{E}_{\eta}^{*} \mathbf{e}_{\eta}^{\downarrow}\right],\right.
$$

the first term of the force (7) takes the form

$$
\begin{aligned}
\frac{1}{2} \operatorname{Re} & \left(\alpha_{e} \nabla\left(\mathbf{E} \mathbf{E}^{*}\right)\right) \\
= & \frac{1}{4} \operatorname{Re}\left(\alpha_{e}\right) \nabla|\mathbf{E}|^{2} \\
& -\frac{1}{2} \operatorname{Im}\left(\alpha_{e}\right) \operatorname{Im}\left[\mathcal{E}_{\xi} \nabla \mathcal{E}_{\xi}^{*}+\mathcal{E}_{\eta} \nabla \mathcal{E}_{\eta}^{*}+\mathcal{E}_{y} \nabla \mathcal{E}_{y}^{*}\right] \\
& +\frac{1}{2}|\mathbf{E}|^{2} \operatorname{Im}\left(\alpha_{e}\right) \nabla \Phi+\operatorname{Im}\left(\alpha_{e}\right) \operatorname{Im}\left(E_{\xi}^{*} E_{\eta}\right) \nabla\left(\mathbf{e}_{\xi} \mathbf{e}_{\eta}^{\downarrow}\right) .
\end{aligned}
$$

In the second term of Eq. (7) we keep only the magnetic gradient force proportional to $\operatorname{Re}\left(\alpha_{m}\right)$. So, the force within a channel in Rayleigh approximation is equal to

$$
\mathbf{F}=\mathbf{F}_{g r}+\mathbf{F}_{\mathrm{pol}}+\mathbf{F}_{\mathrm{ph}}+\mathbf{F}_{\mathrm{curv}}+\mathbf{F}_{\mathrm{m}},
$$

where the electric gradient, polarization, phase, curvilinear, and magnetic gradient forces are given below:

$$
\begin{aligned}
\mathbf{F}_{g r} & =\frac{1}{4} \operatorname{Re}\left(\alpha_{e}\right) \nabla|\mathbf{E}|^{2}, \\
\mathbf{F}_{\mathrm{pol}} & =-\frac{1}{2} \operatorname{Im}\left(\alpha_{e}\right) \operatorname{Im}\left(\mathcal{E}_{\xi} \nabla \mathcal{E}_{\xi}^{*}+\mathcal{E}_{\eta} \nabla \mathcal{E}_{\eta}^{*}+\mathcal{E}_{y} \nabla \mathcal{E}_{y}^{*}\right), \\
\mathbf{F}_{\mathrm{ph}} & =\frac{1}{2}|\mathbf{E}|^{2} \operatorname{Im}\left(\alpha_{e}\right) \nabla \Phi \\
\mathbf{F}_{\mathrm{curv}} & =\operatorname{Im}\left(\alpha_{e}\right) \operatorname{Im}\left(E_{\xi}^{*} E_{\eta}\right) \nabla\left(\mathbf{e}_{\xi} \mathbf{e}_{\eta}\right), \\
\mathbf{F}_{\mathrm{m}} & =\frac{1}{4} \operatorname{Re}\left(\alpha_{m}\right) \nabla|\mathbf{H}|^{2} .
\end{aligned}
$$

The electric and magnetic gradient forces differ by a factor of $s^{2}$ and vanish simultaneously if $|\mathbf{E}|^{2}$ and $|\mathbf{H}|^{2}$ have the same position of extremum. The polarization force is also defined by the gradients of the fields but is much weaker than the gradient force due to the relation $\operatorname{Im} \alpha_{e} \ll \operatorname{Re} \alpha_{e}$. This force takes nonzero values for the nonlinear (circular, elliptic) polarization of the incident wave. The phase force owing to the phase gradient is a common nonconservative force.

The particles can be trapped within the stable channel only by the large electric gradient forces. Hence, the channel is mainly defined by $\nabla|\mathbf{E}|^{2}=0$. Though this condition is satisfied for both the maxima and minima of $|\mathbf{E}|^{2}$, the stable channels correspond to the maxima for the dielectric particles with $\varepsilon>1$ situated in vacuum (this case is interesting for us). The minima of $|\mathbf{E}|^{2}$ can also form stable channels, when the permittivity of the spherical particle is smaller than the permittivity of the ambient medium. The nonconservative forces introduce the corrections to the positions of the channels. These corrections are small and should not affect much the results for the stable channels defined by the intensity maxima.

Thus, the channel position can be found from the set of equations

$$
\frac{\partial|\mathbf{E}|^{2}}{\partial \xi}=0, \quad \frac{\partial|\mathbf{E}|^{2}}{\partial \eta}=0, \quad \frac{\partial|\mathbf{E}|^{2}}{\partial y}=0 .
$$

In the case of the channel equation $\xi(x, z)=$ const, the direction of the channel is specified by the unit vector $\mathbf{e}_{\eta}$, and the forces along the channel can be written as

$$
\begin{aligned}
F_{\mathrm{pol}}= & \mathbf{e}_{\eta} \mathbf{F}_{\mathrm{pol}}=-\frac{1}{2 h_{\eta}} \operatorname{Im}\left(\alpha_{e}\right) \\
& \times \operatorname{Im}\left(\mathcal{E}_{\xi} \frac{\partial \mathcal{E}_{\xi}^{*}}{\partial \eta}+\mathcal{E}_{\eta} \frac{\partial \mathcal{E}_{\eta}^{*}}{\partial \eta}+\mathcal{E}_{y} \frac{\partial \mathcal{E}_{y}^{*}}{\partial \eta}\right), \\
F_{\mathrm{ph}}= & \mathbf{e}_{\eta} \mathbf{F}_{\mathrm{ph}}=\frac{1}{2 h_{\eta}}|\mathbf{E}|^{2} \operatorname{Im}\left(\alpha_{e}\right) \frac{\partial \Phi}{\partial \eta}, \\
F_{\mathrm{curv}}= & \mathbf{e}_{\eta} \mathbf{F}_{\mathrm{curv}}=\frac{1}{h_{\eta}} \operatorname{Im}\left(\alpha_{e}\right) \operatorname{Im}\left(E_{\xi}^{*} E_{\eta}\right) \mathbf{e}_{\xi} \frac{\partial \mathbf{e}_{\eta}}{\partial \eta}, \\
F_{\mathrm{m}}= & \mathbf{e}_{\eta} \mathbf{F}_{\mathrm{m}}=\frac{1}{4 h_{\eta}} \operatorname{Re}\left(\alpha_{m}\right) \frac{\partial|\mathbf{H}|^{2}}{\partial \eta},
\end{aligned}
$$


where $h_{\xi}$ and $h_{\eta}$ are the Lamé coefficients. Having in mind that the quantity

$$
\kappa=\left|\frac{\partial \mathbf{e}_{\eta}}{h_{\eta} \partial \eta}\right|
$$

is the curvature of the curve, we can find the traces of it in the definition of the force $F_{\text {curv }}$. The magnetic gradient force $F_{\mathrm{m}}$ vanishes if $|\mathbf{H}|^{2}=\left|H_{1}\right|^{2}+\left|H_{2}\right|^{2}+\left|H_{3}\right|^{2}$ does not depend on the coordinate along the channel $\eta$. It is feasible, if the field components depend on $\eta$ as $H_{1}=\left|H_{1}\right| \exp \left(\mathrm{i} \phi_{1}(\eta)\right)$, etc. Hence, the magnetic field takes the form $\mathbf{H}=\left|H_{1}\right| \exp \left(\mathrm{i} \phi_{1}(\eta)\right)+$ $\left|H_{2}\right| \exp \left(\mathrm{i} \phi_{2}(\eta)\right)+\left|H_{3}\right| \exp \left(\mathrm{i} \phi_{3}(\eta)\right)$. For instance, such a magnetic field appears in the nondiffractive Bessel and Airy beams.

When the channel is a straight line $\xi=x=$ const, we come to the case of a nondiffracting light beam with constant intensity in the transverse plane. Then $\eta=z$ and the basis vectors coincide with those of the Cartesian coordinates, i.e., $\mathbf{e}_{\xi}=\mathbf{e}_{x}$ and $\mathbf{e}_{\eta}=\mathbf{e}_{z}$. The curvilinear force $F_{\text {curv }}$ obviously turns to zero. Since $\partial \mathcal{E} / \partial z=0$ for the nondiffracting beam and the magnetic energy density $|\mathbf{H}|^{2}$ does not depend on $z$, the polarization force $F_{\text {pol }}$ and the magnetic force $F_{m}$ vanish as well. Only the phase force $F_{\mathrm{ph}}=(1 / 2)|\mathbf{E}|^{2} \operatorname{Im}\left(\alpha_{e}\right) \partial \Phi / \partial z$ controls the particle dynamics in this case.

\section{NONDIFFRACTING LIGHT BEAMS}

\section{A. Nonparaxial Bessel beams}

We consider the well-known nondiffracting light beam formed by the superposition of the plane waves with wave vectors lying on the cone of angle $2 \alpha$ at the vertex-the electromagnetic Bessel beam [21]. If $\alpha$ is great, the Bessel beam is called nonparaxial. In this section, we deal with the nonparaxial transverse electric (TE)-polarized Bessel beams of the order $m$ in vacuum. The electric and magnetic fields in cylindrical coordinates $\xi=r, \eta=\varphi$, and $y$ read [22]

$$
\begin{aligned}
\mathbf{E}= & -\frac{k_{0}}{q} A\left(\mathrm{i} J_{m}^{\prime}(q r) \mathbf{e}_{\varphi}+\frac{\mathrm{m}}{q r} J_{m}(q r) \mathbf{e}_{r}\right) e^{\mathrm{i} m \varphi+\mathrm{i} \beta y}, \\
\mathbf{H}= & A\left(J_{m}(q r) \mathbf{e}_{y}+\frac{\beta}{q}\left(\mathrm{i} J_{m}^{\prime}(q r) \mathbf{e}_{r}-\frac{\mathrm{m}}{q r} J_{m}(q r) \mathbf{e}_{\varphi}\right)\right) \\
& \times e^{\mathrm{i} m \varphi+\mathrm{i} \beta y},
\end{aligned}
$$

where $A$ is the amplitude, $J_{m}$ is the Bessel function of the order $m, J_{m}^{\prime}(x)=d J_{m} / d x$, and $\beta=k_{0} \cos \alpha$ and $q=\sqrt{k_{0}^{2}-\beta^{2}}=$ $k_{0} \sin \alpha$ are the longitudinal and transverse wave numbers, respectively. The picture of the intensity of the Bessel beam is illustrated in Fig. 1(a). The electric field components and phase function equal

$$
\begin{aligned}
\mathcal{E}_{\xi} & =\mathcal{E}_{r}=-\frac{m k_{0}}{q^{2} r} A J_{m}(q r), \\
\mathcal{E}_{\eta} & =\mathcal{E}_{\varphi}=-\frac{i k_{0}}{q} A J_{m}^{\prime}(q r), \\
\Phi & =m \varphi+\beta y .
\end{aligned}
$$
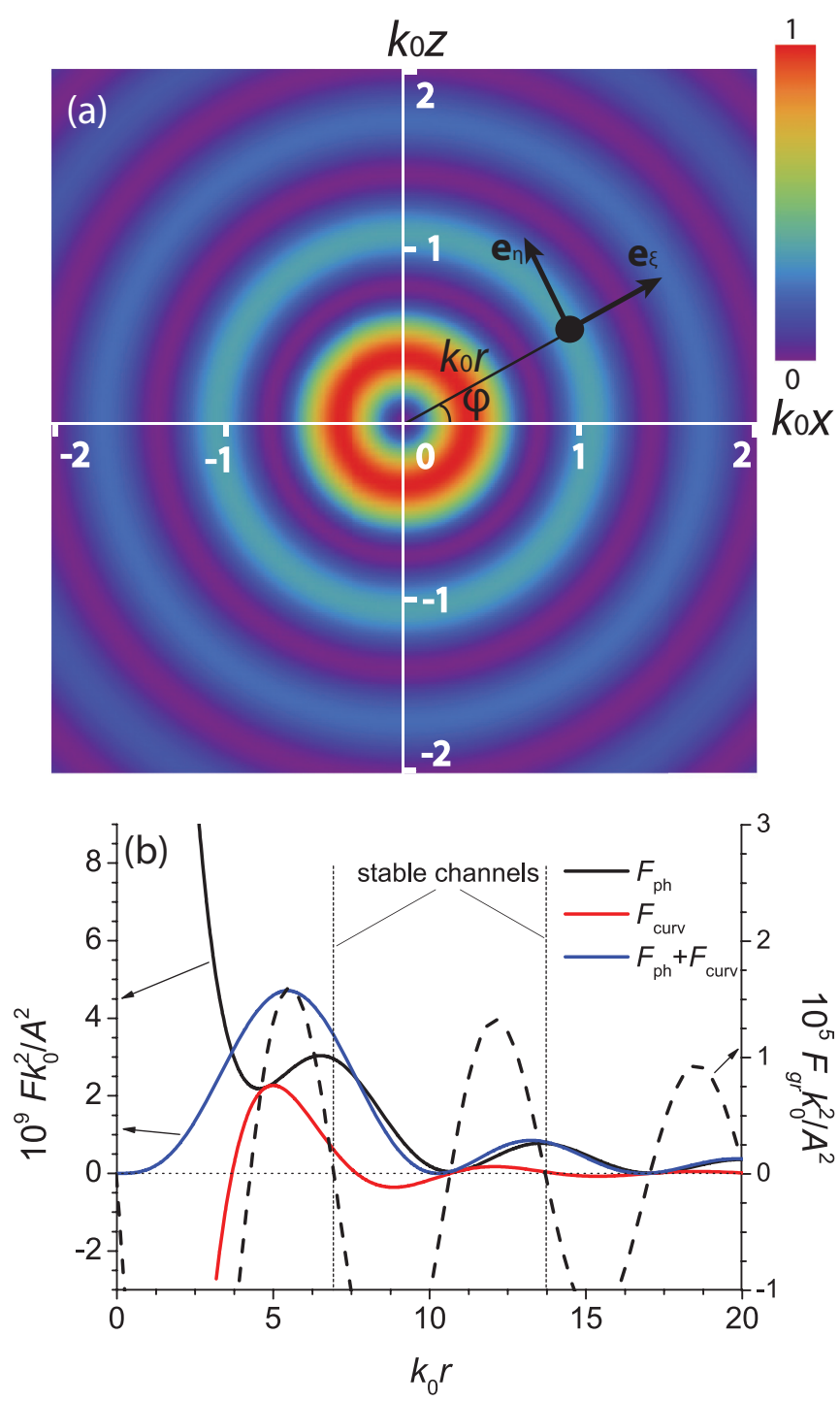

FIG. 1. (Color online) (a) Sketch of the spherical particle in the Bessel beam's cross section $\left(k_{0} x, k_{0} z\right)$. The curvilinear trajectory is the circle $\xi=r=$ const. The particle is trapped by the gradient force and moves in the stable channel due to the nonconservative forces. (b) The gradient and nonconservative optical forces acting on the Rayleigh particle of radius $k_{0} a=0.1$ and dielectric permittivity $\varepsilon=2.25$ in the field of the nonparaxial Bessel beam of the order $m=1\left(q / k_{0}=0.5\right)$.

Since $|\mathbf{E}|^{2}=\left(k_{0} A / q\right)^{2}\left[J_{m}^{\prime 2}+(m / q r)^{2} J_{m}^{2}\right]$ depends only on the coordinate $r$, the channel equation takes the form

$$
\frac{d}{d r}\left(J_{m}^{\prime 2}(q r)+\frac{m^{2}}{q^{2} r^{2}} J_{m}^{2}(q r)\right)=0 .
$$

Maxima of the function $|\mathbf{E}|^{2}$ correspond to the stable channels $r=r_{c}$ or, in other notation, $x^{2}+z^{2}=r_{c}^{2}$. The channels are the cylinders of radius $r_{c}$. The particle is not allowed to move along a curved trajectory, when $r_{c}=0$; therefore, we exclude this case from consideration. We ignore the particle's movement in the straight $y$ direction assuming that it is trapped at $y=0$ by the standing-wave Bessel beam. 
The forces in the channel $r=r_{c}$ can be written as

$$
\begin{aligned}
F_{\mathrm{pol}} & =0, \quad F_{\mathrm{ph}}=\frac{\mathrm{m}}{2 r} \operatorname{Im}\left(\alpha_{e}\right)|\mathbf{E}|^{2}, \\
F_{\text {curv }} & =-\frac{m k_{0}^{2}}{q^{3} r^{2}} A^{2} \operatorname{Im}\left(\alpha_{e}\right) J_{m}(q r) J_{m}^{\prime}(q r), \\
F_{\mathrm{m}} & =\frac{1}{4 r} \operatorname{Re}\left(\alpha_{m}\right) \frac{\partial|\mathbf{H}|^{2}}{\partial \varphi}=0 .
\end{aligned}
$$

The $r$ dependencies of all nonzero forces are depicted in Fig. 1(b). Zero gradient force $F_{g r} \sim d|\mathbf{E}|^{2} / d r$ corresponds to the position of the channel. Half of the channels are stable. They attract the particles; i.e., the gradient force is positive at $r<r_{c}$ and negative at $r>r_{c}$. The stable channels are indicated in Fig. 1(b) by vertical dotted lines.

The curvilinear optical force $F_{\text {curv }}$ can take both positive and negative values. It is always smaller than the phase force $F_{\mathrm{ph}}$; therefore, the net nonconservative force is positive:

$$
\begin{aligned}
F_{\text {ph }}+F_{\text {curv }} \geqslant & \frac{m k_{0}^{2} A^{2}}{q^{3} r^{2}} \operatorname{Im}\left(\alpha_{e}\right) \\
& \times\left|J_{m} J_{m}^{\prime}\right|\left(m-\operatorname{sign}\left(J_{m} J_{m}^{\prime}\right)\right) \geqslant 0,
\end{aligned}
$$

where $\operatorname{sign}\left(J_{m} J_{m}^{\prime}\right)=+1$ for $J_{m} J_{m}^{\prime}>0$ and $\operatorname{sign}\left(J_{m} J_{m}^{\prime}\right)=-1$ for $J_{m} J_{m}^{\prime}<0$. The nonconservative force turns to zero at $m=$ 0 . Thus, the particle moves in the direction governed by the sign of the beam order $m$ (dragged by the orbital momentum of the light beam). Though the curvilinear force cannot be negative in the stable channels of the nonparaxial Bessel light beam of the order $m=1$ [see Fig. 1(b)], $F_{\text {curv }}<0$ for the first channel in the case $m>1$ as shown in Fig. 2.

In fact, from the channel equation (19) we derive

$$
J_{m}^{\prime} J_{m}=\frac{m^{2} J_{m}^{2}+J_{m}^{\prime 2}\left(q r_{c}\right)^{2}}{q r_{c}\left(2 m^{2}-\left(q r_{c}\right)^{2}\right)} .
$$

The curvilinear force in the channel is negative, if $J_{m}^{\prime} J_{m}$ is positive [see Eq. (20)]; i.e., the denominator $2 m^{2}-\left(q r_{c}\right)^{2}>0$ or

$$
k_{0} r_{c}<\frac{\sqrt{2} \mathrm{~m}}{q / k_{0}} .
$$

For $m=1$ [Fig. 1(b)] the curvilinear force in the channel cannot be negative, because the stable channel is out of the domain $k_{0} r_{c}<\sqrt{2} \times 1 / 0.5=2 \sqrt{2}$. However, the first channel position satisfies the conditions $k_{0} r<20 \sqrt{2}$ at $m=2$ and $k_{0} r<30 \sqrt{2}$ at $m=3$. That is why the curvilinear force $F_{\text {curv }}<0$ in the first channel as depicted in Figs. 2(a) and 2(b).

\section{B. Airy beams}

The curvilinear coordinates in the plane $(x, z)$ adopted for the Airy light beam can be written as (see Fig. 3)

$$
\xi=\frac{x}{x_{0}}-\frac{z^{2}}{4 x_{0}^{2}}, \quad \eta=\frac{x}{x_{0}}+2 \ln \left(\frac{z}{x_{0}}\right),
$$

where $x_{0}$ is the scale factor. The basis vectors of the orthogonal coordinate system are equal to

$$
\mathbf{e}_{\xi}=\frac{\mathbf{e}_{x}-\left(z / 2 x_{0}\right) \mathbf{e}_{z}}{\sqrt{1+\left(z / 2 x_{0}\right)^{2}}}, \quad \mathbf{e}_{\eta}=\frac{\mathbf{e}_{z}+\left(z / 2 x_{0}\right) \mathbf{e}_{x}}{\sqrt{1+\left(z / 2 x_{0}\right)^{2}}},
$$
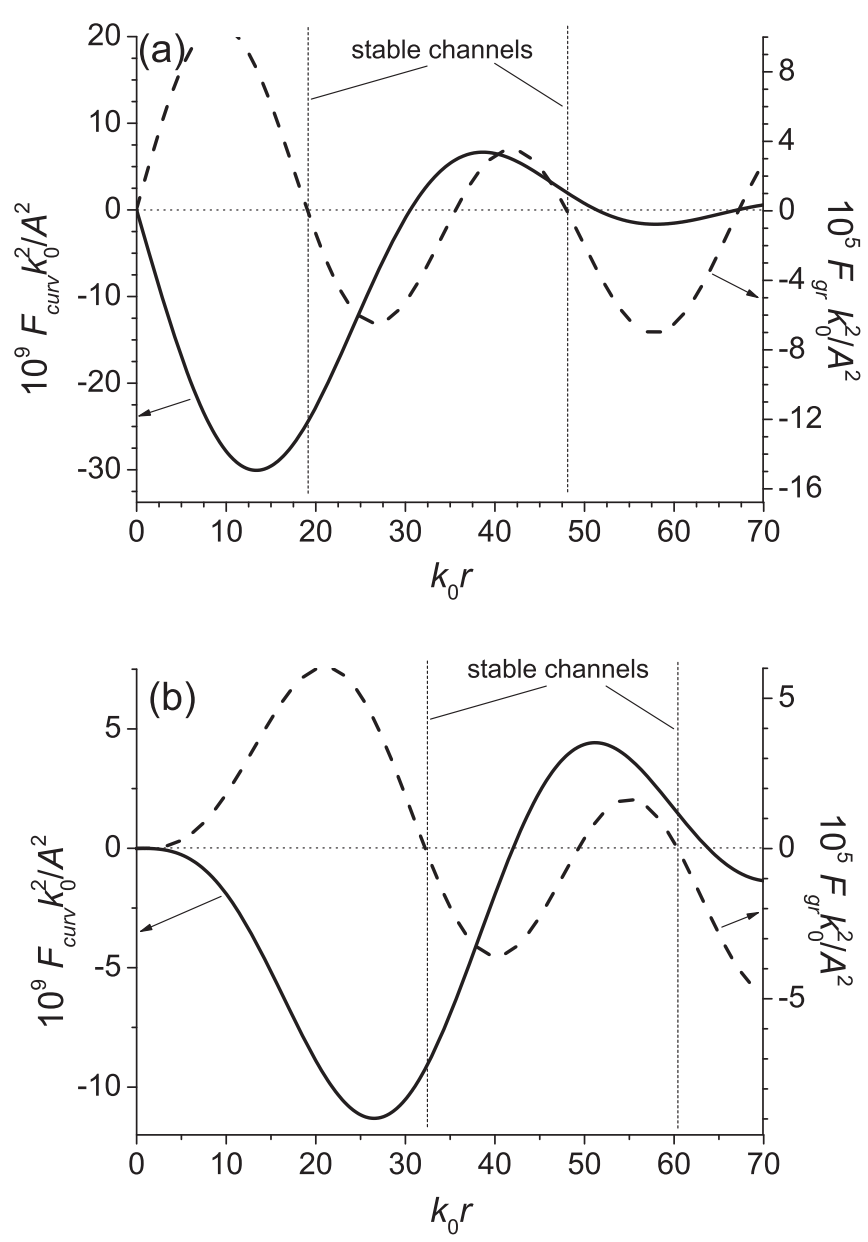

FIG. 2. The curvilinear optical forces in the case of the nonparaxial Bessel beam of the order (a) $m=2$ and (b) $m=3\left(q / k_{0}=0.1\right)$ for the particle's radius $k_{0} a=0.1$ and dielectric permittivity $\varepsilon=2.25$.

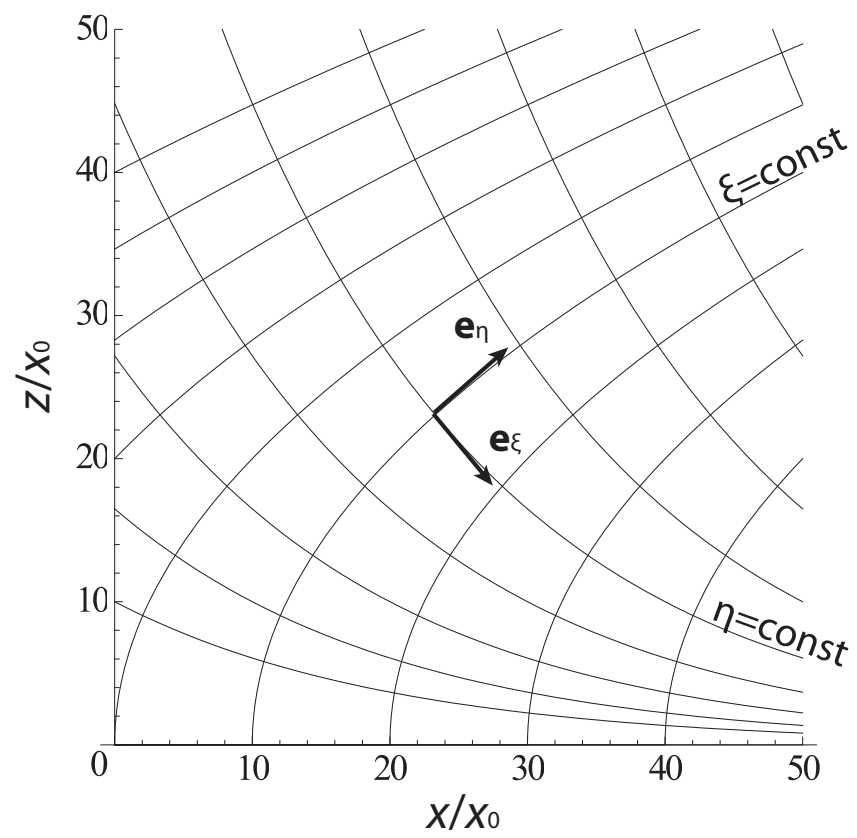

FIG. 3. Coordinate lines $\xi=$ const and $\eta=$ const of the orthogonal curvilinear coordinate system. $\xi=$ const is the trajectory of the Airy beam. 
while the Lamé coefficients take the form

$$
h_{\xi}=\frac{x_{0}}{\sqrt{1+\left(z / 2 x_{0}\right)^{2}}}, \quad h_{\eta}=\frac{z / 2}{\sqrt{1+\left(z / 2 x_{0}\right)^{2}}} .
$$

Using the expression for magnetic field

$$
\mathbf{H}=A_{0} e^{\mathrm{i \Phi}} \operatorname{Ai}(\xi) \mathbf{e}_{y}
$$

as the solution of the paraxial wave equation valid at $k_{0} x_{0} \gg 1$, we derive the electric field from Maxwell's equations:

$$
\begin{aligned}
\mathbf{E}= & \frac{\mathrm{i}}{k_{0}} \nabla \times \mathbf{H}=-\frac{A_{0} e^{\mathrm{i} \Phi}}{\sqrt{1+\left(z / 2 x_{0}\right)^{2}}} \\
& \times\left[\left(A z / 2 x_{0}+B\right) \mathbf{e}_{\xi}+\left(A-B z / 2 x_{0}\right) \mathbf{e}_{\eta}\right],
\end{aligned}
$$

where

$$
\begin{aligned}
\Phi & =k_{0} z-\frac{z^{3}}{12 k_{0}^{3} x_{0}^{6}}+\frac{x z}{2 x_{0}^{2}}, \\
A & =\left(-k_{0}+\frac{z^{2}}{4 k_{0}^{3} x_{0}^{6}}-\frac{x}{2 x_{0}^{2}}\right) \operatorname{Ai}(\xi)-\frac{\mathrm{i} z}{2 x_{0}^{2}} \operatorname{Ai}^{\prime}(\xi), \\
B & =\frac{z}{2 x_{0}^{2}} \operatorname{Ai}(\xi)-\frac{\mathrm{i}}{x_{0}} \operatorname{Ai}^{\prime}(\xi) .
\end{aligned}
$$

Since the magnetic field is written in the paraxial approximation, we should use the same approximation $\left(k_{0} x_{0} \gg 1\right)$ in the expression for the electric field. Then

$$
A \approx-k_{0} \operatorname{Ai}(\xi), \quad B \approx-\frac{\mathrm{i}}{x_{0}} \mathrm{Ai}^{\prime}(\xi), \quad \sqrt{1+\left(z / 2 x_{0}\right)^{2}} \approx 1 .
$$

The vectors $\mathbf{e}_{\xi}$ and $\mathbf{e}_{\eta}$ are oriented approximately along the $z$ and $x$ axes, respectively. Having the above expressions in mind, we make the conclusion that the vector of the gradient force has only a $\xi$ component, because $|\mathbf{E}|^{2}$ does not depend on $\eta$ :

$$
\mathbf{F}_{g r}=\frac{A_{0}^{2}}{4} \operatorname{Re}\left(\alpha_{e}\right) \frac{d}{d \xi}\left(k_{0}^{2} \operatorname{Ai}^{2}(\xi)+\frac{1}{x_{0}^{2}} \operatorname{Ai}^{2}(\xi)\right) \mathbf{e}_{\xi} .
$$

Using the Airy equation, we present the gradient force as

$$
\mathbf{F}_{g r}=\frac{A_{0}^{2} k_{0}^{2}}{2} \operatorname{Re}\left(\alpha_{e}\right) \operatorname{Ai}^{\prime}(\xi) \operatorname{Ai}(\xi)\left(1+\frac{\xi}{k_{0}^{2} x_{0}^{2}}\right) \mathbf{e}_{\xi} .
$$

Then the channels are defined from the condition

$$
\operatorname{Ai}^{\prime}(\xi) \operatorname{Ai}(\xi)\left(1+\frac{\xi}{k_{0}^{2} x_{0}^{2}}\right)=0 .
$$

The guiding forces are directed along the channel which is specified by the unit vector $\mathbf{e}_{\eta}$ :

$$
\begin{aligned}
F_{\mathrm{pol}} & =-\frac{k_{0} A_{0}^{2}}{2 x_{0}^{2}} \operatorname{Im}\left(\alpha_{e}\right) \operatorname{Ai}(\xi) \mathrm{Ai}^{\prime}(\xi), \\
F_{\mathrm{ph}} & =\frac{k_{0} A_{0}^{2}}{2} \operatorname{Im}\left(\alpha_{e}\right)\left(k_{0}^{2} \mathrm{Ai}^{2}(\xi)+\frac{1}{x_{0}^{2}} \operatorname{Ai}^{\prime 2}(\xi)\right), \\
F_{\text {curv }} & =\frac{k_{0} A_{0}^{2}}{2 x_{0}^{2}} \operatorname{Im}\left(\alpha_{e}\right) \operatorname{Ai}(\xi) \operatorname{Ai}^{\prime}(\xi), \\
F_{\mathrm{m}} & =\frac{1}{2 z} \operatorname{Re}\left(\alpha_{m}\right) \frac{\partial \mathrm{Ai}^{2}(\xi)}{\partial \eta}=0 .
\end{aligned}
$$

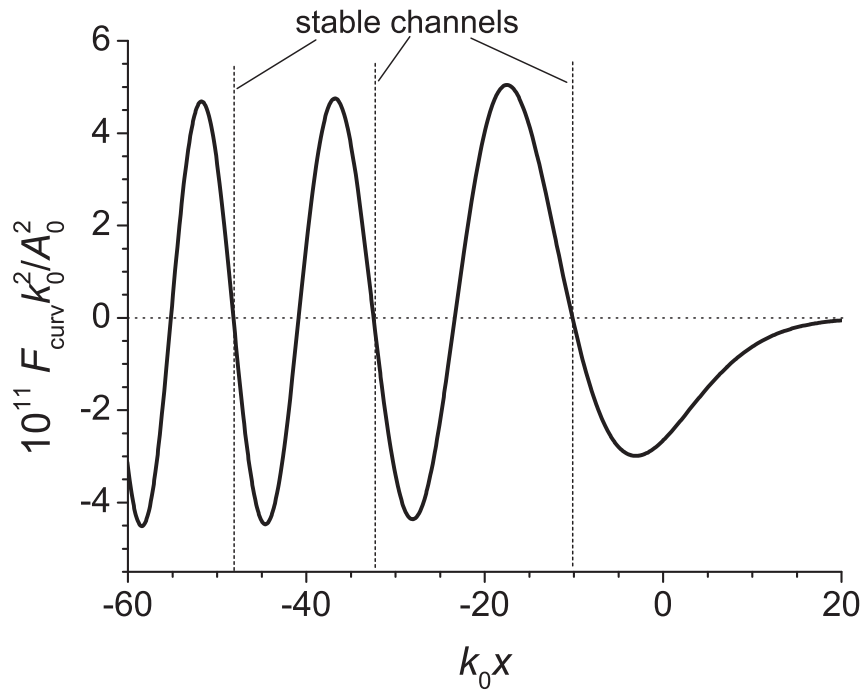

FIG. 4. The curvilinear optical force at $k_{0} z=1$ for the Airy light beam $\left(k_{0} x_{0}=10\right)$. Particle's radius $k_{0} a=0.1$ and dielectric permittivity $\varepsilon=2.25$.

Being nondiffracting, the Airy beam is beneficial for long-distance movement of the particle without a gradient force. In the paraxial regime, the trajectories are close to the straight lines, the curvature is small, and the beam's polarization is almost linear. Therefore, the force owing to the phase gradient is much greater than the other nonconservative (polarization and curvilinear) forces characterized by the factor $1 /\left(k_{0} x_{0}\right)^{2}$. The curvilinear force can take both positive and negative values, though it vanishes within the stable channels (see Fig. 4). It should be noticed that since $F_{\text {curv }}$ is totally compensated by the polarization force $F_{\mathrm{pol}}$, the net nonconservative force $F_{\mathrm{ph}}+F_{\mathrm{pol}}+F_{\text {curv }}=F_{\mathrm{ph}}$ is always positive and the Rayleigh particle cannot be pulled.

\section{ARBITRARY-TRAJECTORY LIGHT BEAMS}

\section{A. Electromagnetic field}

Following Ref. [11], we write the magnetic and electric fields of the beam in vacuum in the plane $(x, z)$ as

$$
\begin{aligned}
\mathbf{H} & =\psi(x, z) \mathbf{e}_{y}-\frac{\mathrm{i}}{k_{0}}\left(\frac{\partial \chi}{\partial x} \mathbf{e}_{z}-\frac{\partial \chi}{\partial z} \mathbf{e}_{x}\right), \\
\mathbf{E} & =\frac{\mathrm{i}}{k_{0}}\left(\frac{\partial \psi}{\partial x} \mathbf{e}_{z}-\frac{\partial \psi}{\partial z} \mathbf{e}_{x}\right)+\chi(x, z) \mathbf{e}_{y},
\end{aligned}
$$

where the functions $\psi$ and $\chi$ specify the solutions in the form of transverse magnetic (TM)- and TE-polarized electromagnetic beams, respectively.

The function $\psi$ (the same is valid for $\chi$ ) satisfies the Helmholtz wave equation

$$
\left(\frac{\partial^{2} \psi}{\partial x^{2}}+\frac{\partial^{2} \psi}{\partial z^{2}}\right)+k_{0}^{2} \psi=0 .
$$

The solution of this equation can be represented in the form [11]

$$
\psi(x, z)=\frac{\mathrm{i} k_{0}}{2} \int_{-\infty}^{\infty} A\left(x^{\prime}\right) e^{\mathrm{i} \phi\left(x^{\prime}\right)} \frac{z}{r} H_{1}^{(1)}\left(k_{0} r\right) d x^{\prime},
$$


where $r\left(x, z, x^{\prime}\right)=\sqrt{\left(x-x^{\prime}\right)^{2}+z^{2}}$ and $H_{m}^{(1)}\left(k_{0} r\right)$ is the $m$ th-order Hankel function of the first kind. The amplitude $A\left(x^{\prime}\right)$ and phase $\phi\left(x^{\prime}\right)$ describe the field $\psi(x, 0)=$ $A(x) \exp (\mathrm{i} \phi(x))$ at the "initial" plane $z=0$. For great arguments of the Hankel function $H_{1}^{(1)}$ the asymptotic behavior is $H_{1}^{(1)}\left(k_{0} r\right) \sim \exp \left(\mathrm{i} k_{0} r\right) / \sqrt{k_{0} r}$. Therefore, the phase under the integral equals $k_{0} r+\phi=k_{x}\left(x-x^{\prime}\right)+k_{z} z+\phi$, where $k_{x}$ and $k_{z}\left(k_{x}\right)=\sqrt{k_{0}^{2}-k_{x}^{2}}$ are the projections of the wave vector.

The phase function $\phi\left(x^{\prime}\right)$ controls the form of the channel's trajectory to guide a particle and can be determined using the stationary phase approximation. After differentiation of the stationary phase equation

$$
k_{x}\left(x-x^{\prime}\right)+k_{z} z+\phi\left(x^{\prime}\right)=\mathrm{const}
$$

over $x^{\prime}$

$$
-k_{x}+\frac{d \phi\left(x^{\prime}\right)}{d x^{\prime}}=0
$$

and over $k_{x}$,

$$
x-x^{\prime}+\frac{d k_{z}}{d k_{x}} z=0
$$

we arrive at the expression for the wave number $k_{x}$,

$$
k_{x}=\frac{d \phi\left(x^{\prime}\right)}{d x^{\prime}}=\phi^{\prime},
$$

and at the ray equation

$$
x=x^{\prime}-k_{z}^{\prime}\left(\phi^{\prime}\right) z
$$

Here $k_{z}^{\prime}=d k_{z} / d k_{x}$.

On the other hand, the channel in the plane $(x, z)$ is described by the equation $x_{c}=f\left(z_{c}\right)$. Then the ray equation of the channel is equal to

$$
x^{\prime}-k_{z}^{\prime}\left(\phi^{\prime}\right) z_{c}=f\left(z_{c}\right) .
$$

Differentiating this equation over $z_{c}$, we get the relation between the ray trajectory and initial phase $\phi\left(x^{\prime}\right)$ :

$$
f^{\prime}\left(z_{c}\right)=-k_{z}^{\prime}\left(\phi^{\prime}\right)
$$

or

$$
f^{\prime}\left(z_{c}\right)=\frac{\phi^{\prime}}{\sqrt{k_{0}^{2}-\phi^{\prime 2}}},
$$

where $f^{\prime}=d f / d z$ is the derivative. Thus, the phase equals

$$
\phi\left(x^{\prime}\right)=\int \frac{k_{0} f^{\prime}\left(z_{c}\left(x^{\prime}\right)\right)}{\sqrt{1+f^{\prime 2}\left(z_{c}\left(x^{\prime}\right)\right)}} d x^{\prime} .
$$

The dependence of $z_{c}$ on $x^{\prime}$ required in Eq. (46) can be deduced from Eq. (43) as

$$
f\left(z_{c}\right)=x^{\prime}+f^{\prime}\left(z_{c}\right) z_{c} .
$$

Summing up, the electric and magnetic fields (35) with an arbitrary trajectory $x_{c}=f\left(z_{c}\right)$ are described by a couple of scalar functions $\psi$ and $\chi$. These functions are the solutions (37) which form the set of stable channels $x_{c}=f\left(z_{c}\right)$, if the phase $\phi\left(x^{\prime}\right)$ is governed by Eq. (46).

\section{B. Curvilinear coordinates}

To use the previously derived formulas for the optical forces, it is necessary to introduce the orthogonal curvilinear coordinates. One of the coordinates defines the trajectory as $\xi=$ const. The couple of curvilinear coordinates in the $(x, z)$ plane reads

$$
\xi=x-f(z), \quad \eta=x+\int \frac{d z}{f^{\prime}(z)} .
$$

Taking the partial derivatives of the coordinates (48) over $\xi$ and $\eta$, we derive the relationships

$$
\begin{array}{ll}
\frac{\partial x}{\partial \xi}=\frac{1}{1+f^{\prime 2}(z)}, & \frac{\partial z}{\partial \xi}=-\frac{f^{\prime}(z)}{1+f^{\prime 2}(z)}, \\
\frac{\partial x}{\partial \eta}=\frac{f^{\prime 2}(z)}{1+f^{\prime 2}(z)}, & \frac{\partial z}{\partial \eta}=\frac{f^{\prime}(z)}{1+f^{\prime 2}(z)} .
\end{array}
$$

The Lamé coefficients are of the form

$$
\begin{aligned}
& h_{\xi}=\sqrt{\left(\frac{\partial x}{\partial \xi}\right)^{2}+\left(\frac{\partial z}{\partial \xi}\right)^{2}}=\frac{1}{\sqrt{1+f^{\prime 2}(z)}}, \\
& h_{\eta}=\sqrt{\left(\frac{\partial x}{\partial \eta}\right)^{2}+\left(\frac{\partial z}{\partial \eta}\right)^{2}}=\frac{\left|f^{\prime}(z)\right|}{\sqrt{1+f^{\prime 2}(z)}} .
\end{aligned}
$$

The basis vectors of the curvilinear coordinates equal

$$
\begin{aligned}
& \mathbf{e}_{\xi}=\frac{1}{h_{\xi}}\left(\frac{\partial x}{\partial \xi} \mathbf{e}_{x}+\frac{\partial z}{\partial \xi} \mathbf{e}_{z}\right)=\frac{\mathbf{e}_{x}-f^{\prime}(z) \mathbf{e}_{z}}{\sqrt{1+f^{\prime 2}(z)}}, \\
& \mathbf{e}_{\eta}=\frac{1}{h_{\eta}}\left(\frac{\partial x}{\partial \eta} \mathbf{e}_{x}+\frac{\partial z}{\partial \eta} \mathbf{e}_{z}\right)=\frac{f^{\prime}}{\left|f^{\prime}\right|} \frac{\mathbf{e}_{z}+f^{\prime}(z) \mathbf{e}_{x}}{\sqrt{1+f^{\prime 2}(z)}},
\end{aligned}
$$

while Cartesian basis vectors can be written as

$$
\mathbf{e}_{x}=\frac{\mathbf{e}_{\xi}+\left|f^{\prime}(z)\right| \mathbf{e}_{\eta}}{\sqrt{1+f^{\prime 2}(z)}}, \quad \mathbf{e}_{z}=\frac{f^{\prime}}{\left|f^{\prime}\right|} \frac{\mathbf{e}_{\eta}-\left|f^{\prime}(z)\right| \mathbf{e}_{\xi}}{\sqrt{1+f^{\prime 2}(z)}} .
$$

By substituting these vectors into the equation for the electric field (35) we obtain

$$
\begin{aligned}
\mathbf{E}= & \frac{\mathrm{i}}{k_{0} \sqrt{1+f^{\prime 2}(z)}}\left[-\left(\frac{\partial \psi}{\partial x} f^{\prime}(z)+\frac{\partial \psi}{\partial z}\right) \mathbf{e}_{\xi}\right. \\
& \left.+\frac{f^{\prime}(z)}{\left|f^{\prime}(z)\right|}\left(\frac{\partial \psi}{\partial x}-f^{\prime}(z) \frac{\partial \psi}{\partial z}\right) \mathbf{e}_{\eta}\right]+\chi \mathbf{e}_{y} .
\end{aligned}
$$

Using Eq. (48), the coordinates $x$ and $z$ can be expressed by means of the curvilinear coordinates $\xi$ and $\eta$. Then $\mathbf{E}$ is written entirely in the curvilinear coordinates.

\section{Optical forces}

In this section, we write the optical forces acting on the particle in the field of the accelerating beam in question. The electric gradient force

$$
\mathbf{F}_{g r}=\frac{1}{4 k_{0}^{2}} \operatorname{Re}\left(\alpha_{e}\right) \nabla\left(\left|\frac{\partial \psi}{\partial x}\right|^{2}+\left|\frac{\partial \psi}{\partial z}\right|^{2}+k_{0}^{2}|\chi|^{2}\right)
$$

can be neglected only in the diffraction-free channels defined as

$$
\nabla\left(\left|\frac{\partial \psi}{\partial x}\right|^{2}+\left|\frac{\partial \psi}{\partial z}\right|^{2}+k_{0}^{2}|\chi|^{2}\right)=0
$$


However, the light beams under consideration are not nondiffracting ones. The electric gradient force does not vanish except at the point where $|\mathbf{E}|^{2}$ takes an extreme value.

The magnetic gradient force in the channel's direction is much smaller than the electric gradient force in the Rayleigh approximation and equals

$$
\begin{aligned}
F_{m} & =\mathbf{e}_{\eta} \mathbf{F}_{m} \\
& =\frac{1}{4 k_{0}^{2} h_{\eta}} \operatorname{Re}\left(\alpha_{m}\right)\left[k_{0}^{2} \frac{\partial|\psi|^{2}}{\partial \eta}+\frac{\partial}{\partial \eta}\left|\frac{\partial \chi}{\partial x}\right|^{2}+\frac{\partial}{\partial \eta}\left|\frac{\partial \chi}{\partial z}\right|^{2}\right] .
\end{aligned}
$$

There are three types of nonconservative forces. We present the curvilinear optical force as

$$
F_{\text {curv }}=-\frac{\kappa}{k_{0}^{2}} \frac{f^{\prime \prime}}{\left|f^{\prime \prime}\right|} \frac{f^{\prime}}{\left|f^{\prime}\right|} \operatorname{Im}\left(\alpha_{e}\right) \operatorname{Im}\left(\frac{\partial \psi}{\partial x} \frac{\partial \psi^{*}}{\partial z}\right),
$$

where

$$
\kappa=\frac{\left|f^{\prime \prime}(z)\right|}{\left(1+f^{\prime 2}(z)\right)^{3 / 2}}
$$

is the curvature of the trajectory. It should be noted that the curvilinear optical force is defined by the TM polarization of the beam (function $\psi$ ) and the curvature. The direction of the force depends on the sign of the first and second derivatives of the curve function $f$.

The explicit function of the phase is not specified in the definition of the electric field; hence, we are not able to divide the polarization and phase forces. Their sum can be written as

$$
\begin{aligned}
F_{\mathrm{ph}}+F_{\mathrm{pol}}= & -\frac{1}{2 h_{\eta}} \operatorname{Im}\left(\alpha_{e}\right) \\
& \times \operatorname{Im}\left(E_{\xi} \frac{\partial E_{\xi}^{*}}{\partial \eta}+E_{\eta} \frac{\partial E_{\eta}^{*}}{\partial \eta}+E_{y} \frac{\partial E_{y}^{*}}{\partial \eta}\right) .
\end{aligned}
$$

By substituting the field components we arrive at the following expression:

$$
\begin{aligned}
F_{\mathrm{ph}}+ & F_{\mathrm{pol}} \\
= & -\frac{\operatorname{Im}\left(\alpha_{e}\right)}{2 k_{0}^{2} h_{\eta}} \operatorname{Im}\left(\frac{\partial \psi}{\partial x} \frac{\partial^{2} \psi^{*}}{\partial \eta \partial x}+\frac{\partial \psi}{\partial z} \frac{\partial^{2} \psi^{*}}{\partial \eta \partial z}+k_{0}^{2} \chi \frac{\partial \chi^{*}}{\partial \eta}\right) \\
& -F_{\text {curv }}
\end{aligned}
$$

The net nonconservative force is equal to

$$
\begin{aligned}
F_{\mathrm{ph}} & +F_{\mathrm{pol}}+F_{\mathrm{curv}} \\
= & -\frac{\operatorname{Im}\left(\alpha_{e}\right)}{2 k_{0}^{2} h_{\eta}} \operatorname{Im}\left(\frac{\partial \psi}{\partial x} \frac{\partial^{2} \psi^{*}}{\partial \eta \partial x}+\frac{\partial \psi}{\partial z} \frac{\partial^{2} \psi^{*}}{\partial \eta \partial z}+k_{0}^{2} \chi \frac{\partial \chi^{*}}{\partial \eta}\right) .
\end{aligned}
$$

\section{Example: Circular trajectory}

In the case of the circular trajectory described by the equation $x_{c}=f\left(z_{c}\right)=\sqrt{R^{2}-z_{c}^{2}}$, the channel ray equation (47) takes the form

$$
\sqrt{R^{2}-z_{c}^{2}}=x^{\prime}-\frac{z_{c}^{2}}{\sqrt{R^{2}-z_{c}^{2}}} .
$$

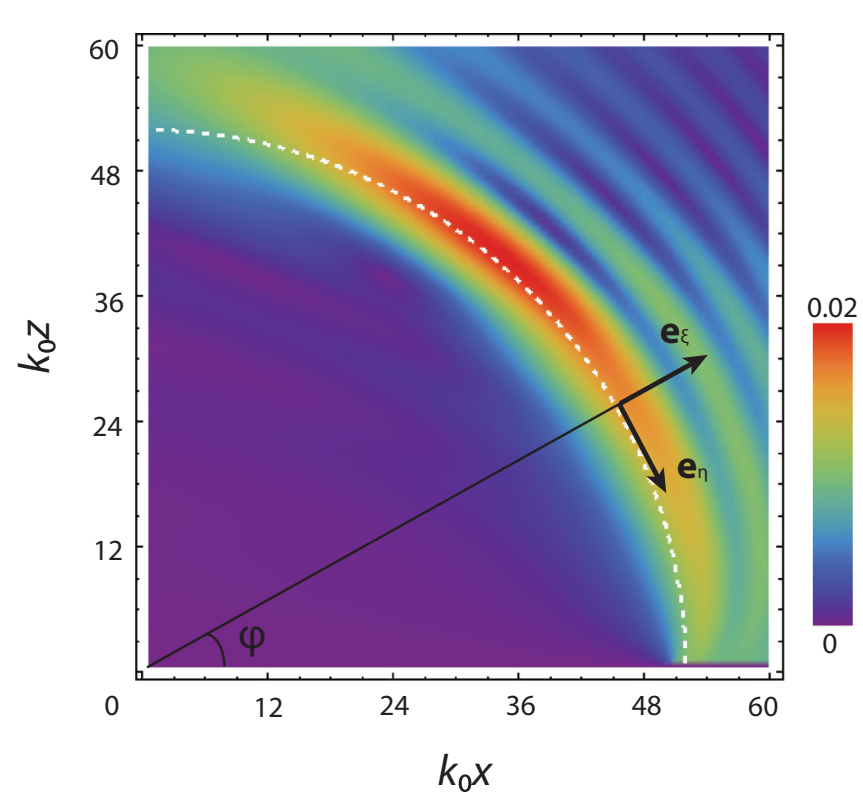

FIG. 5. (Color online) Dimensionless function $|\psi(x, z)| / A_{0}$ with the circular caustic of radius $k_{0} R=50(\gamma=1)$. The optical forces in Fig. 6 are calculated along the dashed line of radius $k_{0} R_{1}=52$.

The solution of this equation establishes the dependence of $z_{c}$ on $x^{\prime}$ as follows:

$$
z_{c}\left(x^{\prime}\right)=\frac{R}{x^{\prime}} \sqrt{x^{\prime 2}-R^{2}} .
$$

Then the phase (46) of the field at the initial plane equals

$$
\phi\left(x^{\prime}\right)=-k_{0} R \sqrt{x^{\prime 2} / R^{2}-1}+k_{0} R \arccos \left(R / x^{\prime}\right) .
$$

If $\left|x^{\prime}\right|>R$, the wave is propagating (phase is a real number). If $\left|x^{\prime}\right|<R$, the phase is complex and the wave is evanescent; hence, its contribution is negligible. One can choose the amplitude as in Ref. [11]: $A\left(x^{\prime}\right)=A_{0} x^{\prime-\gamma} H\left(\left|x^{\prime}\right|-\right.$ $R$ ), where $\gamma$ is the constant, and $H(x)$ is the Heaviside step function $(H(x)=1$ for $x>0$ and $H(x)=0$ for $x<0)$. However, the Heaviside step function is not necessary here, because the domain $\left|x^{\prime}\right|<R$ contributes little owing to the evanescent character of waves.

The dependence of the function $\psi$ ( $y$ component of the magnetic field $\mathbf{H}$ ) on coordinates is demonstrated in Fig. 5. The trajectory is indeed close to the quarter of the circle though some deviations occur. These are connected with the approximate treatment of the considered accelerating beams based on the use of the Hankel function asymptotics. The field is not nondiffracting, but it possesses the gradient in both directions $\mathbf{e}_{\eta}$ and $\mathbf{e}_{\xi}$.

All gradient optical forces discussed in the paper are shown in Fig. 6(a) for the case of a TM-polarized accelerating beam $(\chi=0)$ : two projections of the electric gradient force $F_{\xi}=$ $\mathbf{F}_{g r} \mathbf{e}_{\xi}$ and $F_{\eta}=\mathbf{F}_{g r} \mathbf{e}_{\eta}$ as well as the magnetic gradient force $F_{m}=\mathbf{F}_{m} \mathbf{e}_{\eta}$. If $F_{\xi}=0$, the electric gradient force is tangent to the circle. However, this occurs only at some angles $\phi$. The gradient forces $F_{\eta}$ and $F_{m} \ll F_{\eta}$ vanish at the same point, which corresponds to the intensity maximum on the circle. The nonconservative forces in Fig. 6(b) can be divided into two parts: the great phase-polarization force and small curvilinear 

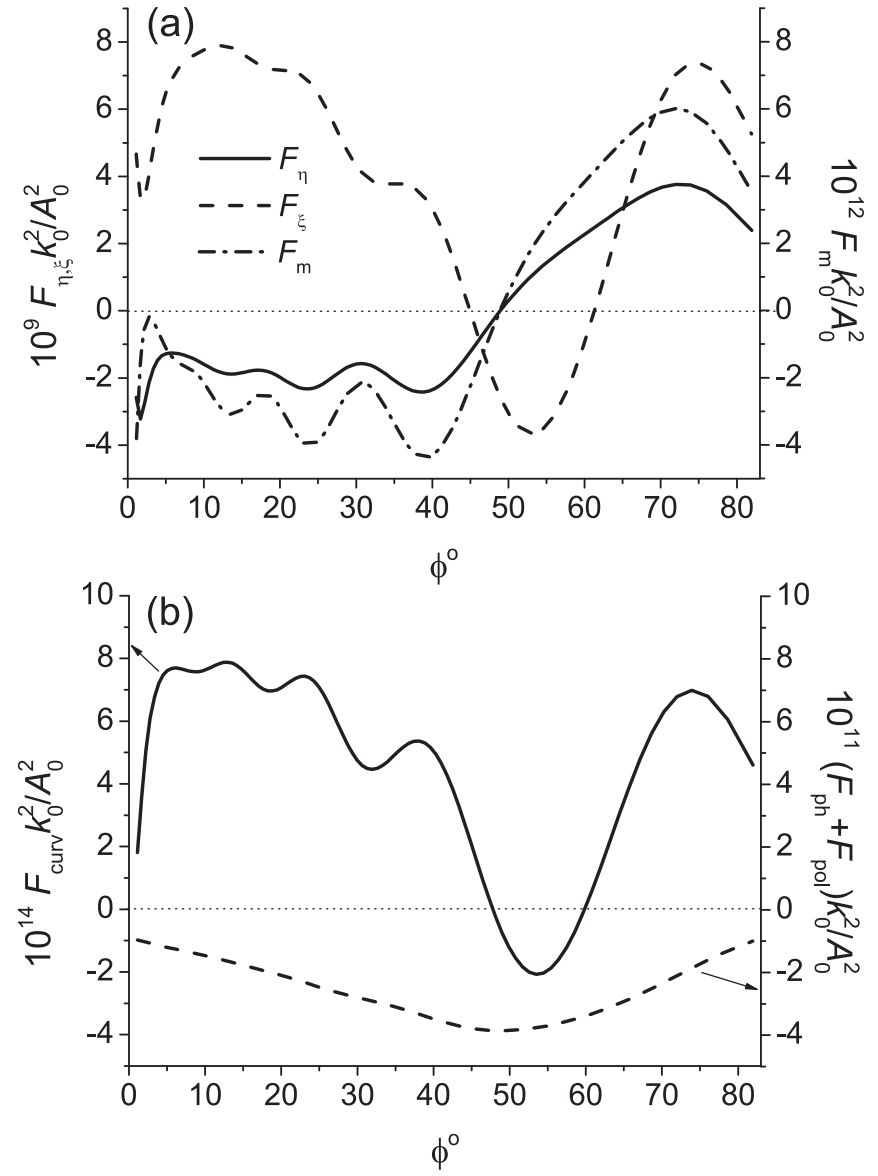

FIG. 6. (a) The gradient and (b) nonconservative optical forces at the circular trajectory of radius $k_{0} R_{1}=52$ (see the dashed line in Fig. 5). The particle radius is $k_{0} a=0.1$ and dielectric permittivity is $\varepsilon=2.25$.

one. Thus, for the given electromagnetic beam, we can rank the forces as follows: $F_{\xi} \sim F_{\eta}>F_{\text {ph }}+F_{\text {pol }}>F_{m}>F_{\text {curv }}$. The force due to the curvilinear beam's trajectory $F_{\text {curv }}$ is negligible. Its dependence on the angle $\phi$ is similar to that of the gradient force $F_{\xi}$, but not the same. It can be expected that, in the channel defined by $F_{\xi}=0$, the curvilinear force should decrease.

The curvilinear force can be enlarged due to the increase of the curvature $\kappa=R^{-1}$ of the curve $x=f(z)$, i.e., to the decrease of the radius $R$ of the circular trajectory. When $k_{0} R \sim 1$, the curvilinear force is comparable in magnitude with the sum of phase and polarization forces. However, in this case the diffraction plays an important part and the method of the light beam composition considered in the current section does not provide the curvilinear trajectory. Nevertheless, the model of the nondiffracting Bessel beam as an exact solution of the Maxwell equations is applicable even for the small trajectory radii $R$ (notice that the forces $F_{\text {curv }}$ and $F_{\text {ph }}$ are comparable in Fig. 1).

\section{CONCLUSION AND OUTLOOK}

We have discussed the types of optical forces acting on the nonmagnetic spherical particles calculated in the Rayleigh approximation. We take into account the gradient force owing to the magnetic polarizability of the particle $\mathbf{F}_{\mathrm{m}}$ and the nonconservative force $\mathbf{F}_{\text {curv }}$ due to the accelerating behavior of the field. The magnetic gradient force is comparable with the nonconservative force and, therefore, cannot be neglected in general.

In this paper we only propose the concept of curvilinear optical forces and demonstrate its application to several examples. The curvilinear force is proportional to the curvature of the trajectory of the accelerating beam. Hence, it is stronger on the steeper parts of trajectories. The curvilinear force cannot be omitted, because it can be of the same order of magnitude as the traditional nonconservative force produced by the phase gradient. To illustrate how the curvilinear force can be increased, one can consider the bigger particles. If we limit ourselves by the dipolar approximation, which is valid until the effects of the quadrupole moments are negligible, we can generalize the results of the Rayleigh approximation quite easily. The size parameters in dipolar approximation are up to $s \sim 1.5$ [23]. The polarizabilities of dipolar particles are described by the Mie-theory-based formulas Eq. (2) and the types of the optical forces are determined from Eq. (1). The further derivation of the optical forces is straightforward, leading to the electric and magnetic curvilinear forces. We expect that the curvilinear optical forces can be used in optical micromanipulation by the accelerating beams.

\section{ACKNOWLEDGMENTS}

Dr. Denis V. Novitsky is acknowledged for help in numerical calculations and critical reading of the manuscript. The author is grateful for the Grant of the President of the Republic of Belarus for financial support.
[1] G. A. Siviloglou, J. Broky, A. Dogariu, and D. N. Christodoulides, Phys. Rev. Lett. 99, 213901 (2007).

[2] G. A. Siviloglou and D. N. Christodoulides, Opt. Lett. 32, 979 (2007).

[3] M. V. Berry and N. L. Balazs, Am. J. Phys. 47, 264 (1979).

[4] A. V. Novitsky and D. V. Novitsky, Opt. Lett. 34, 3430 (2009).

[5] L. Carretero, P. Acebl, S. Blaya, C. Garcia, A. Fimia, R. Madrigal, and A. Murciano, Opt. Express 17, 22432 (2009).

[6] A. Torre, Opt. Commun. 283, 4146 (2010).
[7] I. Kaminer, R. Bekenstein, J. Nemirovsky, and M. Segev, Phys. Rev. Lett. 108, 163901 (2012).

[8] Y. Hu, P. Zhang, C. Lou, S. Huang, J. Xu, and Z. Chen, Opt. Lett. 35, 2260 (2010).

[9] I. D. Chremmos, Z. Chen, D. N. Christodoulides, and N. K. Efremidis, Opt. Lett. 37, 5003 (2012).

[10] I. D. Chremmos and N. K. Efremidis, Phys. Rev. A 88, 063816 (2013).

[11] R.-S. Penciu, V. Paltoglou, and N. K. Efremidis, Opt. Lett. 40, 1444 (2015). 
[12] E. Greenfield, M. Segev, W. Walasik, and O. Raz, Phys. Rev. Lett. 106, 213902 (2011).

[13] J. Rogel-Salazar, H. A. Jimenez-Romero, and S. Chavez-Cerda, Phys. Rev. A 89, 023807 (2014).

[14] J. Baumgartl, M. Mazilu, and K. Dholakia, Nat. Photonics 2, 675 (2008).

[15] H. Cheng, W. Zang, W. Zhou, and J. Tian, Opt. Express 18, 20384 (2010).

[16] Y. Jiang, K. Huang, and X. Lu, Opt. Express 21, 24413 (2013).

[17] Y. Yang, W.-P. Zang, Z.-Y. Zhao, and J.-G. Tian, Opt. Express 20, 25681 (2012).
[18] Y. Yang, W.-P. Zang, Z.-Y. Zhao, and J.-G. Tian, Opt. Express 21, 6186 (2013).

[19] M. Nieto-Vesperinas, J. J. Saenz, R. Gomez-Medina, and L. Chantada, Opt. Express 18, 11428 (2010).

[20] C. F. Bohren and D. R. Huffman, Absorption and Scattering of Light by Small Particles (Wiley, New York, 1998).

[21] J. Durnin, J. Opt. Soc. Am. A 4, 651 (1987).

[22] A. V. Novitsky and D. V. Novitsky, J. Opt. Soc. Am. A 24, 2844 (2007).

[23] A. Garcia-Etxarri, R. Gomez-Medina, L. S. Froufe-Perez, C. Lopez, L. Chantada, F. Scheffold, J. Aizpurua, M. NietoVesperinas, and J. J. Saenz, Opt. Express 19, 4815 (2011). 